\author{
Marquette University \\ e-Publications@Marquette
}

Electrical and Computer Engineering Faculty Research and Publications

Electrical and Computer Engineering,

Department of

$9-2-2011$

\title{
Stochastically Resilient Observer Design for a Class of Continuous-Time Nonlinear Systems
}

Chung Seop Jeong

Marquette University

Edwin E. Yaz

Marquette University, edwin.yaz@marquette.edu

Yvonne I. Yaz

Milwaukee School of Engineering

Follow this and additional works at: https://epublications.marquette.edu/electric_fac

Part of the Computer Engineering Commons, and the Electrical and Computer Engineering Commons

\section{Recommended Citation}

Jeong, Chung Seop; Yaz, Edwin E.; and Yaz, Yvonne I., "Stochastically Resilient Observer Design for a Class of Continuous-Time Nonlinear Systems" (2011). Electrical and Computer Engineering Faculty Research and Publications. 12.

https://epublications.marquette.edu/electric_fac/12 


\title{
Stochastically Resilient Observer Design for a Class of Continuous-Time Nonlinear Systems
}

\author{
Chung Seop Jeong*. Edwin Engin Yaz.*Yvonne Ilke Yaz ** \\ * Marquette University, Department of Electrical and Computer Engineering, Milwaukee, Wisconsin 53201,USA \\ (Tel: 414-288-6820; e-mail: chung.jeong@mu.edu,edwin.yaz@mu.edu). \\ ** Milwaukee School of Engineering, Math Department, Milwaukee, Wisconsin 53202, USA \\ (e-mail:yaz@msoe.edu)
}

\begin{abstract}
This work addresses the design of stochastically resilient or non-fragile continuous-time Luenberger observers for systems with incrementally conic nonlinearities. Such designs maintain the convergence and/or performance when the observer gain is erroneously implemented due possibly to computational errors i.e. round off errors in computing the observer gain or changes in the observer parameters during operation. The error in the observer gain is modeled as a random process and a common linear matrix inequality formulation is presented to address the stochastically resilient observer design problem for a variety of performance criteria. Numerical examples are given to illustrate the theoretical results.
\end{abstract}

Keywords: Nonlinear observer and filter design; Robust estimation; Lyapunov methods

\section{INTRODUCTION}

An observer whose performance deteriorates considerably or diverges easily by a small perturbation in the observer gain is referred to as a "fragile" or "non-resilient" observer. Resilience refers to the insensitivity to gain changes whereas robustness usually implies insensitivity to plant or design parameters. The concept of fragility of controllers is introduced in (Keel and Bhattacharyya, 1997). It is shown that even vanishingly small perturbations in the control coefficients may destabilize the closed-loop system. After the publication of (Keel and Bhattacharyya, 1997), the subject of fragility has gained more attention. A quadratic optimal state feedback controller that is resilient against perturbations in control gain is proposed in (Jadbabaie, et al., 1998). In (Dorato, 1998), an overview of the resilient design techniques is presented. The resilience of control systems in digital implementation of a continuous time controller design is investigated in (Keel and Bhattacharyya, 1998). The synthesis of a resilient regulator for linear systems is described in (Famularo, et al., 1998). In (Takabashi, et al., 2000), the design of robust non-fragile state feedback controllers with controller gains in a given polytope is addressed. Robust non-fragile Kalman filter design for a class of linear systems with norm-bounded uncertainties and multiplicative uncertainties in the filter gain is given in (Yang and Wang, 2001). Resilient filtering for a class of linear continuous-time systems with norm-bounded uncertainties and multiplicative and additive perturbations is investigated in (Mahmoud, 2004).

In the present paper, different approach to resilience is taken for the continuous-time models in that the observer gain perturbation is stochastic in nature. In this sense, it is the non-trivial continuous-time nonlinear extension of the stochastically resilient discrete-time observer design paper by the authors (Yaz, et al., 2006). A novel design of stabilityand performance- resilient observers is introduced in continuous-time for a variety of performance criteria. Process and measurement disturbances are of finite-energy type and the observer gain perturbations are modeled as a broadband noise process. Various design formulations are expressed in a general linear matrix inequality (LMI) (Boyd, et al., 1994) framework. This result is a natural follow up to the result for linear models in (Jeong, et al., 2007). This result is also the extension of (Jeong, et al., 2008) to the stochastically resilient case. Some illustrative examples are also included to depict the feasibility regions of the design LMI and the change in estimation error behaviour based on different performance criteria used in design.

The following notation is used in this work: $x \in R^{n}$ denotes an n-dimensional vector with real elements and with the associated norm $\|x\|=\left(x^{T} x\right)^{1 / 2}$ where $(\cdot)^{T}$ represents the transpose. $A \in R^{m \times n}$ denotes an $m \times n$ matrix with real elements. $A^{-1}$ is the inverse of matrix A, $A>0(A<0)$ means $A$ is a positive (negative) definite matrix, and $\mathrm{I}_{\mathrm{m}}$ is an identity matrix of dimension m. $\lambda_{\text {min }}(A)\left(\lambda_{\text {max }}(A)\right)$ denotes the minimum(maximum) eigenvalue of the symmetric matrix A. E $\{\mathrm{x}\}$ denotes the expectation of x. $L_{2}$ is the space of all vector disturbances $w(t)$ with finite energy $\int_{0}^{\infty}\|w(t)\|^{2} d t<\infty$.

\section{SIGNAL AND ERROR DYNAMICS}

Consider the continuous-time nonlinear system 


$$
\frac{d x}{d t}=f(x, u, w)
$$

and the linear measurement equation

$$
y=C x+D u+G w
$$

where $x \in R^{m}$ is the state to be estimated from the knowledge of the measurement $y \in R^{p}$ and control input $u \in R^{n} . w$ is an $L_{2}$ disturbance input.

The nonlinear vector function $f$ is assumed to be a measurable function of its arguments, and it satisfies the following incrementally conic condition

$$
\begin{aligned}
\|\mathscr{e}(\tilde{x}, w)\| \equiv \| f\left(x_{1}, u, w\right)- & f\left(x_{2}, u, 0\right)-\left[A\left(x_{1}-x_{2}\right)+F w\right] \| \\
\leq & \left\|C_{f}\left(x_{1}-x_{2}\right)+D_{f} w\right\|
\end{aligned}
$$

for some matrices $A, F, C_{f}$, and $\mathrm{D}_{f}$, and $x_{1}, x_{2}, \tilde{x}=x_{1}-x_{2} \in R^{n}$.

Note that the Lipschitz condition is a special case of the condition (1-c) where $A=0, \quad F=0, \quad C_{f}=\alpha I \quad(\alpha>0), \quad D_{f}=0$ resulting in

$$
f\left(x_{1}, u, w\right)-f\left(x_{2}, u, 0\right)\|\leq \alpha\| x_{1}-x_{2} \|
$$

Consider also the formal representation (Arnold, 1974), (Gard, 1988) of a stochastic state observer in the Luenberger form:

$$
\frac{d \hat{x}}{d t}=f(\hat{x}, u, 0)+(K+\Delta(t))(y-C \hat{x}-D u)
$$

where $\Delta(t)$ represents the time-varying error either in computing the observer gain $K$ or during the operation of the observer. In this work, a formal stochastic description of the error in the filter gain is given as follows:

$$
\Delta(t)=\sum_{i=1}^{N} \frac{d \alpha_{i}(t)}{d t} K^{i}
$$

where $\frac{d \alpha_{i}(t)}{d t}$ are standard (zero mean, and unit variance), normally distributed, mutually uncorrelated, scalar, white noise processes and $K^{i}$ are known perturbation matrices. The zero mean property chosen for the gain perturbation represents the realistic situation where the perturbations can be positive or negative in an equally likely manner. The general time varying property is attributed to the gain perturbations by assuming $\frac{d \alpha_{i}(t)}{d t}$ as a random process because this allows models where, in addition to an original computational error mode in the gain, different amounts of perturbations that may occur during operation. This is the generalized form of the random constant perturbation model, which has been used in robustness studies involving structured parameter perturbations (Yaz, 1995).

Let $e \triangleq x-\hat{x}$ denote the state estimation error. Substituting from equation (1-a), (1-b) and (2), we find that the error dynamics obey the following Ito differential equation (Gard, 1988):

$$
\begin{aligned}
d e & =f(x, u, w)-f(\hat{x}, u, 0) \pm(A e+F w) \\
& +K[C e+G w] d t+\sum_{i=1}^{N} K^{i}(C e+G w) d \alpha_{i}
\end{aligned}
$$

where $\alpha_{i}(i=1, . ., N)$ are mutually uncorrelated standard Wiener processes. Note that on (4), the third term on the right hand side is added and subtracted, where $A$ and $F$ are defined in (1-c).

\section{PERFORMANCE CRITERIA}

Let $\mathrm{z}$ denote the performance output where

$$
z=C_{z} e+D_{z} w
$$

Consider the stochastic differential inequality $L V+\delta\|z\|^{2}+\varepsilon\|w\|^{2}-\beta z^{T} w<0$

for a $V(t)=e^{T}(t) P e(t)$ where $P>0$ and L denoting the infinitesimal generator (Arnold, 1974), (Gard, 1988) of (4). It will be later shown that satisfaction of this inequality will make it possible to realize various design objectives. For the chosen $\mathrm{V}$, this yields:

$$
\begin{aligned}
L V= & 2[(A-K C) e+(F-K G) w]^{T} P e+2 \mathfrak{F}^{T}(e, w) P e \\
& +\sum_{i=1}^{N}(C e+G w)^{T} K^{i T} P K^{i}(C e+G w) \\
& \leq 2[(A-K C) e+(F-K G) w]^{T} P e \\
& +\sum_{i=1}^{N}(C e+G w)^{T} K^{i T} P K^{i}(C e+G w) \\
& +\gamma\left(C_{f} e+D_{f} w\right)^{T}\left(C_{f} e+D_{f} w\right)+\gamma^{-1} e^{T} P^{2} e
\end{aligned}
$$

for $\gamma>0$, because

$2 \mathfrak{F}^{T}(e, w) P e \leq \gamma\left\|\mathscr{J}^{2}\right\|^{2}+\gamma^{-1} e^{T} P^{2} e$

$\leq \gamma\left(C_{f} e+D_{f} w\right)^{T}\left(C_{f} e+D_{f} w\right)+\gamma^{-1} e^{T} P^{2} e$

after using (1-c).

Let us now interpret inequality (6). Substituting $V(t)$ into (6), integrating and taking the expectation using stochastic calculus (Arnold, 1974), (Gard, 1988) gives

$$
\begin{aligned}
& E\left\{e(t)^{T} P e(t)\right\} \leq E\left\{e(0)^{T} P e(0)\right\} \\
& \quad-E\left\{\int_{0}^{t}\left(\delta\|z(\tau)\|^{2}+\varepsilon\|w(\tau)\|^{2}-\beta z(\tau)^{T} w(\tau)\right) d \tau\right\}
\end{aligned}
$$

or the generalized performance criteria

$$
\begin{aligned}
& \lambda_{\text {min }}(P) E\left\{\|e(t)\|^{2}\right\}<\lambda_{\text {max }}(P) E\left\{\|e(0)\|^{2}\right\} \\
& -E\left\{\int_{0}^{t}\left(\delta\|z\|^{2}+\varepsilon\|w\|^{2}-\beta z^{T} w\right) d \tau\right\} \\
& \text { by using Rayleigh's inequality }
\end{aligned}
$$

$\left(\lambda_{\min }(P)\|e\|^{2} \leq e^{T} P e \leq \lambda_{\max }(P)\|e\|^{2}\right.$ ) twice, that will be shown to allow several optimization formulations possible in a unified eigenvalue problem (Boyd, et al., 1994) framework. 
First of all, we take $F=0, G=0$, and $D_{z}=0$ to eliminate the additive disturbance dependence. In this case, if we let $\delta=\varepsilon=0$, and $\beta=0$, (8b) yields

$$
E\left\{\|e(t)\|^{2}\right\}<\frac{\lambda_{\text {max }}(P)}{\lambda_{\text {min }}(P)} E\left\{\|e(0)\|^{2}\right\}
$$

This means that by minimizing $\lambda_{\max }(P)$ and maximizing $\lambda_{\min }(P)$, we can lower the bound on the mean square (m.s.) value of the estimation error.

For m.s. asymptotic stability, we take $\delta>0, \beta=0$, and $\varepsilon=0$ with $\mathrm{C}_{\mathrm{z}}$ square and nonsingular, to yield $\lambda_{\min }(P) E\left\{\|e(t)\|^{2}\right\}$

$$
<\lambda_{\min }(P)\left\{\|e(t)\|^{2}\right\}+\delta E\left\{\int_{0}^{t}\|z(\tau)\|^{2} d \tau\right\}<\lambda_{\max }(P) E\left\{\left\|e_{0}\right\|^{2}\right\}
$$

so

$$
E\left\{\|e(t)\|^{2}\right\}<\frac{\lambda_{\text {max }}(P)}{\lambda_{\text {min }}(P)} E\left\{\|e(0)\|^{2}\right\}
$$

Therefore, minimizing $\lambda_{\max }(P)$ and maximizing $\lambda_{\min }(P)$ will result in a faster rate of decrease of error to zero for asymptotic stability.

For the same choice of parameter matrices except for an arbitrary $\mathrm{C}_{\mathrm{z}}$, taking $\delta>0, \beta=0$, and $\varepsilon=0$, (8b) will yield a bound on the energy of the performance output in terms of the initial m.s. estimation error $e(0)$ :

$$
E\left\{\int_{0}^{t}\|z(\tau)\|^{2} d \tau\right\}<\frac{1}{\delta} \lambda_{\max }(P) E\left\{\|e(0)\|^{2}\right\}
$$

Minimizing $\lambda_{\max }(P)$ and maximizing $\delta$ will give us a smaller bound on the energy of the performance output. This is a m.s. $\mathrm{H}_{2}$ observer.

In the case of additive disturbance $w$, and for nonzero values of $F, G$, and $D_{z}$, by setting $\delta=1, \beta=0$, and $\varepsilon<0$ for $e(0)=0$, gives the result

$$
E\left\{\int_{0}^{t}\|z(\tau)\|^{2} d \tau\right\}<-\in \int_{0}^{t}\|w(\tau)\|^{2} d \tau
$$

which means a bound on the m.s. $L_{2}$ to $L_{2}$ gain of the estimator, or a $H_{\infty}$ design.

Again when $e(0)=0$, if we use this formulation, we can design several m.s. dissipative observers by using different values of $\delta, \beta$, and $\varepsilon$. All of these cases will require the choice $D_{z}+D_{z}{ }^{T}>0$ in the performance output in (5).

For example, taking $\delta=0, \beta=1$, and $\varepsilon=0$ will give m.s. passivity

$$
E\left\{\int_{0}^{t} z(\tau)^{T} w(\tau) d \tau\right\}>0
$$

If we take $\delta=0, \beta=1$, and $\varepsilon>0$, it will yield the m.s. input strict passivity result:

$$
E\left\{\int_{0}^{t} z(\tau)^{T} w(\tau) d \tau\right\}>\varepsilon \int_{0}^{t}\|w(\tau)\|^{2} d \tau
$$

If we set $\delta>0, \beta=1$, and $\varepsilon=0$, we will get m.s. output strict passivity:

$$
E\left\{\int_{0}^{t} z(\tau)^{T} w(\tau) d \tau\right\}>\delta E\left\{\int_{0}^{t}\|z(\tau)\|^{2} d \tau\right\}
$$

M.s. very strict passivity, which is the m.s. passivity both in the terms of the input and the output, will be obtained if we set $\delta>0, \beta=1$, and $\varepsilon>0$ :

$$
\begin{aligned}
& E\left\{\int_{0}^{t} z(\tau)^{T} w(\tau) d \tau\right\} \\
& \quad>\varepsilon \int_{0}^{t}\|w(\tau)\|^{2} d \tau+\delta E\left\{\int_{0}^{t}\|z(\tau)\|^{2} d \tau\right\}
\end{aligned}
$$

As described above, the LMI formulation to be given below enables us to design various observers according to different performance criteria in a common framework.

\section{LMI FORMULATION}

The non-noisy and noisy cases will be treated separately in the following development. First, consider inequality (6) with $F=0, G=0, D_{z}=0$, and $\varepsilon=\beta=0$. Inequality (7) yields

$$
\begin{gathered}
e^{T}\left[2(A-K C)^{T} P+\sum_{i=1}^{N} C^{T} K^{i T} P K^{i} C\right. \\
\left.+\delta C_{z}{ }^{T} C_{z}+\gamma C_{f}{ }^{T} C_{f}+\gamma^{-1} P^{2}\right] e<0
\end{gathered}
$$

Using the Schur's complement (Boyd, et al., 1994) for the matrix in brackets yields

$$
e^{T} Q e>0
$$

for

$$
Q=\left[\begin{array}{ll}
q_{11} & q_{12} \\
q_{12}^{T} & q_{22}
\end{array}\right]>0
$$

where

$$
\begin{aligned}
q_{11}= & -P A+Y C-A^{T} P+C^{T} Y^{T} \\
& -\sum_{i=1}^{N} C^{T} K^{i T} P K^{i} C-\delta C_{z}{ }^{T} C_{z}-\gamma C_{f}^{T} C_{f} \\
q_{12}= & P, q_{22}=\gamma I
\end{aligned}
$$

with $Y=P K$. Therefore, we have:

Theorem 1. Let (16) hold for $P>0, \gamma>0$, and $Y$. Then, for $\delta=0$, this implies inequality (9a) or m.s. error boundedness. For $\delta>0$, this implies (9b) for $C_{z}$ square and nonsingular or m.s. asymptotic convergence and (10) or m.s. $H_{2}$ performance for an arbitrary $\mathrm{C}_{\mathrm{z}}$. The necessary resilient observer gain $K$ is found by $K=P^{-1} Y$.

In the noisy case, similar arguments will lead to 


$$
\begin{aligned}
& e^{T} P[(A-K C) e+(F-K G) w] \\
& +[(A-K C) e+(F-K G) w]^{T} P e \\
& +\gamma\left(C_{f} e+D_{f} w\right)^{T}\left(C_{f} e+D_{f} w\right)+\gamma^{-1} e^{T} P^{2} e \\
& +\sum_{i=1}^{N}\left((C e+G w)^{T} K^{i T} P K^{i}(C e+G w)\right. \\
& +\delta\left(C_{z} e+D_{z} w\right)^{T}\left(C_{z} e+D_{z} w\right) \\
& +\varepsilon w^{T} w-\beta\left(C_{z} e+D_{z} w\right)^{T} w<0
\end{aligned}
$$

Using the Schur's complement result (Boyd, et al., 1994), (17) is found to be equivalent to

$$
S=\left[\begin{array}{ccc}
s_{11} & s_{12} & s_{13} \\
S_{12}^{T} & s_{22} & s_{23} \\
s_{13}^{T} & s_{23}^{T} & s_{33}
\end{array}\right]>0
$$

for

$$
\begin{aligned}
s_{11}= & -P A+Y C-A^{T} P+C^{T} Y^{T} \\
& -\sum C^{T} K^{i T} P K^{i} C-\delta C_{z}^{T} C_{z}-\gamma C_{f}^{T} C_{f} \\
s_{12}= & -P F+Y G \\
& -\sum C^{T} K^{i T} P K^{i} G-\delta C_{z}^{T} D_{z}+\frac{\beta}{2} C_{z}^{T}-\gamma C_{f}^{T} D_{f} \\
s_{22}= & -\sum G^{T} K^{i T} P K^{i} G \\
& -\varepsilon I-\delta D_{z}^{T} D_{z}+\frac{\beta}{2}\left(D_{z}+D_{z}^{T}\right)-\gamma D_{f}^{T} D_{f} \\
s_{13}= & P, s_{23}=0, s_{33}=\gamma I
\end{aligned}
$$

So, in the noisy case, we have:

Theorem 2. Let the LMI (18) hold for $P>0, \gamma>0$, and $Y$. Then for different choice of design parameters $\delta, \beta$, and $\varepsilon$, the inequalities (11)-(15) hold (i.e. $H_{\infty}$ and various m.s. passivity criteria are satisfied). The necessary resilient observer gain $K$ is found from $K=P^{-1} Y$.

\section{NUMERICAL STUDIES}

Example 1. This example shows the feasibility regions in the $\mathrm{P}$ and Y coordinates for LMIs (16) and (18) for a onedimensional system and various design parameters. After finding values for $\mathrm{Y}$ and $\mathrm{P}$, the corresponding resilient observer gains can be found from $K=P^{-1} Y$. The design parameters are given in Table 1. for two different performance indices m.s. $H_{2}$ and m.s. input strict passivity.

\begin{tabular}{|c|c|c|}
\hline & $H_{2}$-Observer & Input Strict Passivity \\
\hline$A$ & 0.5 & 0.5 \\
\hline$C$ & 1 & 1 \\
\hline$C_{z}$ & 1 & 1 \\
\hline$D_{z}$ & 0 & 1 \\
\hline$C_{f}$ & 1 & 1 \\
\hline
\end{tabular}

\begin{tabular}{|c|c|c|}
\hline$D_{f}$ & 0 & 1 \\
\hline$F$ & 0 & 1 \\
\hline$G$ & 0 & 0.1 \\
\hline$K^{i}$ & 1 & 1 \\
\hline$\gamma$ & 1 & 3 \\
\hline$\delta$ & $0.1,1,3,5$ & 0 \\
\hline$\beta$ & 0 & 1 \\
\hline$\varepsilon$ & 0 & $0.1,0.5,0.7,0.9$ \\
\hline
\end{tabular}

Table 1. Design parameter values

The corresponding areas are shaded differently to indicate how the shape of the regions changes as the design parameters change. Large areas should be interpreted as containing the small areas inside.

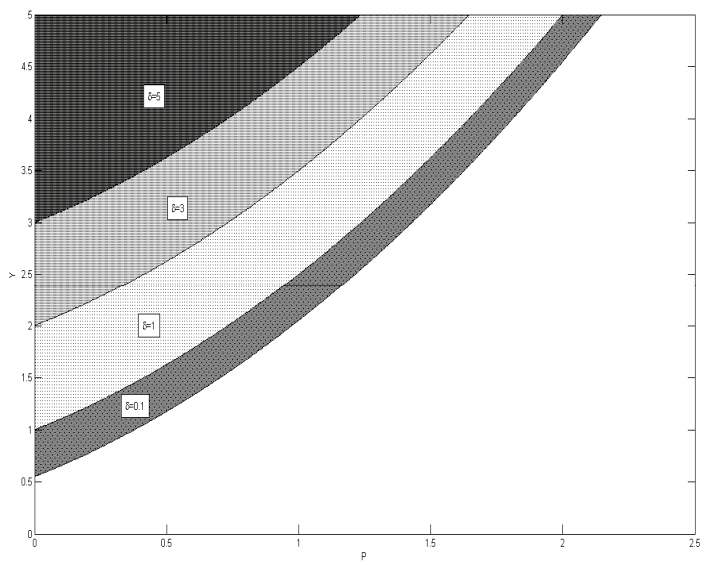

Fig. 1. M.s. $H_{2}$ observer feasibility regions

Fig. 1 shows how the feasibility region for the $H_{2}$ sub optimal resilient observer gets smaller as $\delta$ increases, as expected. This is because it becomes more difficult to satisfy the bound on the output energy which keeps getting smaller in (10).

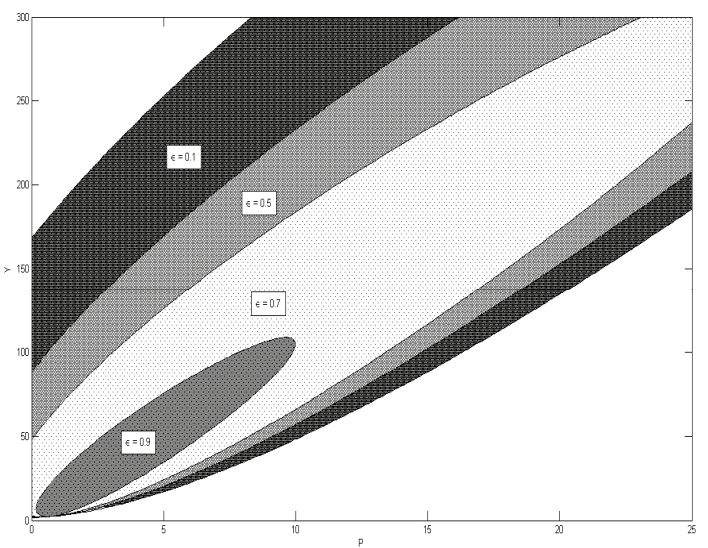

Fig. 2. M.s. input strictly passive observer feasibility regions

Fig. 2. shows the feasibility region of the input strictly passive resilient observer. As $\varepsilon$ increases from 0.1 to 0.9 , the feasibility region becomes smaller as expected. This is 
because the dissipation rate increases with $\varepsilon$ and it becomes more difficult to satisfy (13) with increasing $\varepsilon$.

Example 2. This example contains a comparative study of the error responses of observers for various performance criteria. Consider the following unstable nonlinear system

$$
\left[\begin{array}{l}
\dot{x}_{1} \\
\dot{x}_{2}
\end{array}\right]=\left[\begin{array}{ll}
0 & 1 \\
0 & 0
\end{array}\right]\left[\begin{array}{l}
x_{1} \\
x_{2}
\end{array}\right]+\left[\begin{array}{l}
0 \\
-\sin x_{1}
\end{array}\right]+F w, y=\left[\begin{array}{ll}
1 & 0
\end{array}\right] x+G w
$$

which is simulated with the resilient observer using randomly generated (standard normally distributed white noise) $\Delta(t)$ to test the resilience of our design. A first-order discretized equivalent (Gard, 1988), with the sampling period $\mathrm{T}=0.01$ $\mathrm{sec}$, of the stochastically perturbed estimation error equation is used in simulations. The m.s. $\mathrm{H}_{2}$ observer (for $w(t) \equiv 0, t \geq 0$ ), m.s. input strict passivity, and m.s. output strict passivity were chosen to show the differences in response for these different performance criteria using the proposed design methodology.

Table 2. shows the design parameters used in simulation studies.

\begin{tabular}{|c|c|c|c|}
\hline & $\begin{array}{c}H_{2^{-}} \\
\text {Observer }\end{array}$ & $\begin{array}{c}\text { Input Strict } \\
\text { Passivity }\end{array}$ & $\begin{array}{c}\text { Output Strict } \\
\text { Passivity }\end{array}$ \\
\hline$C_{z}$ & {$[11]$} & {$[11]$} & {$[11]$} \\
\hline$D_{z}$ & 1 & 1 & 1 \\
\hline$C_{f}$ & 0.5 & 0.7 & 0.7 \\
\hline$D_{f}$ & 0 & 0 & 0 \\
\hline$F$ & 0 & {$[1 ; 1]$} & {$[1 ; 1]$} \\
\hline$G$ & 0 & 0.2 & 0.2 \\
\hline$K^{i}$ & {$[1 ; 1]$} & {$[1 ; 1]$} & {$[1 ; 1]$} \\
\hline$\delta$ & 1 & 0 & 0.5 \\
\hline$\beta$ & 0 & 1 & 1 \\
\hline$\varepsilon$ & 0 & 0.5 & 0 \\
\hline$w(t)$ & 0 & $5 \cdot(0.99)^{\mathrm{t}}$ & $5 \cdot(0.99)^{\mathrm{t}}$ \\
\hline$\left\|\mathrm{e}_{0}\right\|$ & 0.14 & 0 & 0 \\
\hline
\end{tabular}

Table 2. Design parameter values

For the simulation of the m.s. $\mathrm{H}_{2}$ observer, the observer gain is found to be $K=[1.7027 ; 1.5 \quad 85]$. For the case of the input strict passivity, $\mathrm{K}$ is found to be $K=[5.5907 ; 5.5416]$, and the gain $K$ for the output strict passivity case is found to be $K=[6.0669 ; 6.0724]$. Each simulation result was averaged over 30 runs. The states variables and estimation error norm plots for each case vs. time in seconds are given in Fig.s 3-5. Notice the change in the response magnitude and shape for different criteria. Fig. 6 is the comparison of the norm of the error for the same three performance criteria. The $\mathrm{H}_{2}$-Observer seems to produce the fastest response due to the absence of the external disturbance.
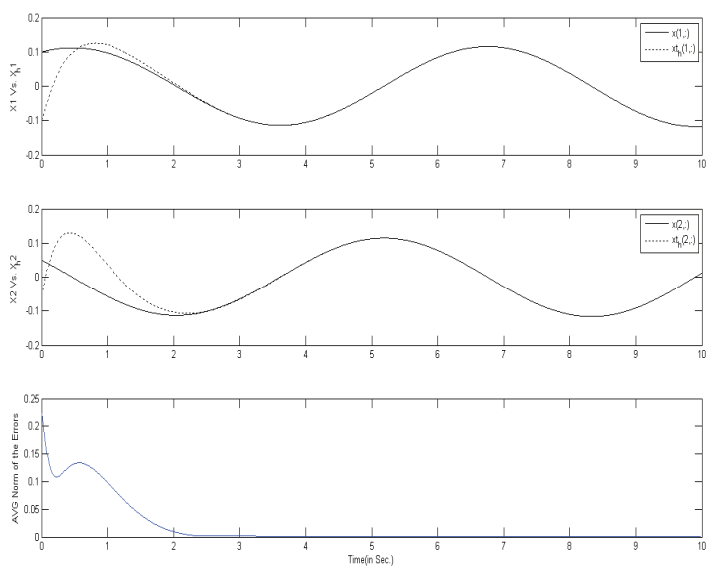

Fig. 3. Plot of States and $\|e(t)\|$ for the m.s. $H_{2}$ observer
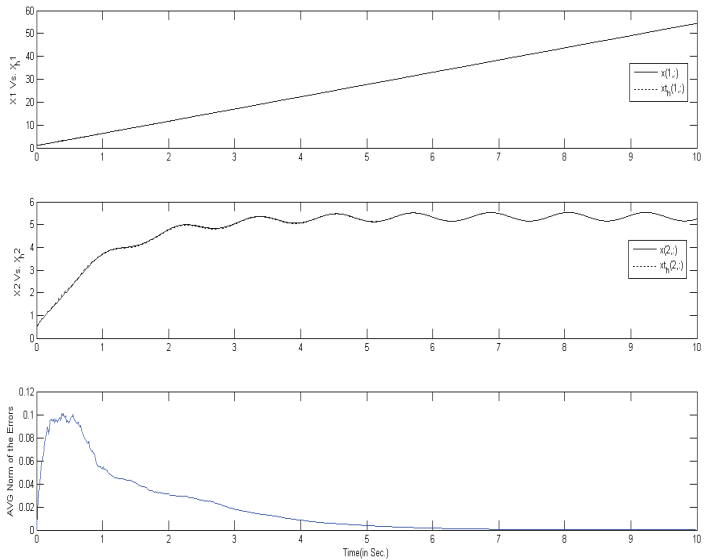

Fig. 4. Plot of States and $\|e(t)\|$ for the Input strict passivity
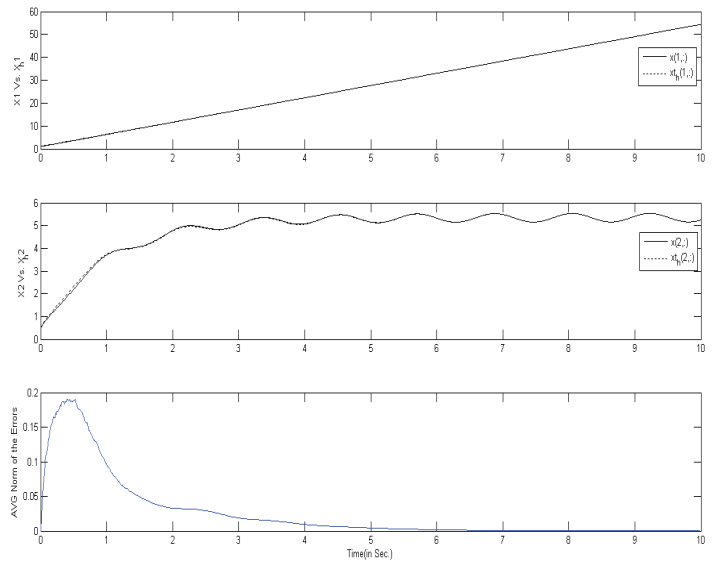

Fig. 5. Plot of States and $\|e(t)\|$ for the Output strict passivity 


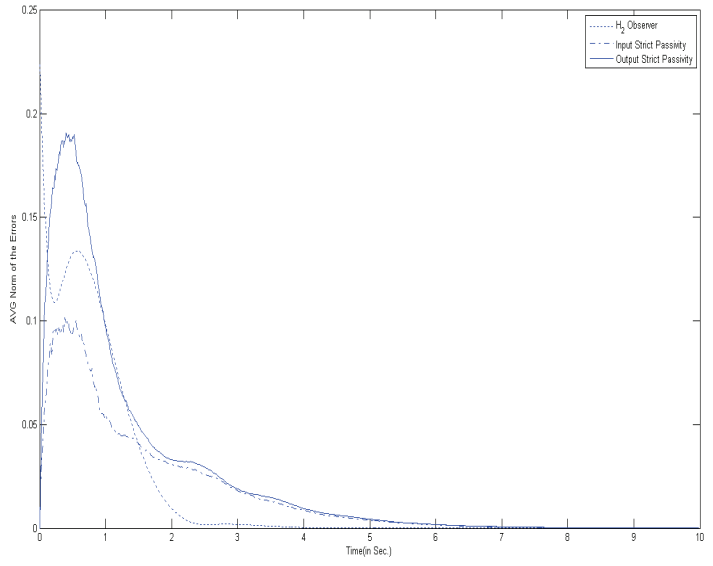

Fig. 6. Comparison of $\|e(t)\|$ for the Different Performance Criteria

\section{CONCLUSIONS}

This paper has presented a simple solution to the problem of stochastically non-fragile or resilient observer design for continuous-time nonlinear systems with finite energy type additive disturbances where the observer gain is randomly perturbed due possibly to computational errors or changes during operation. An LMI based approach has been proposed to design observers with guaranteed stochastic performance and/or stability and the theoretical results introduced have been accompanied by numerical studies.

\section{REFERENCES}

Arnold, L. (1974). Stochastic Differential Equations: Theory and Applications, Wiley, NY.

Boyd, S., ElGhaoui, L., Feron, E., and Balakrishnan, V. (1994). Linear Matrix Inequalities in System and Control Theory, SIAM Studies in Applied Mathematics. SIAM, Philadelphia.

Dorato, P. "Non-fragile controller design: an overview," (1998). "Proc. of American Control Conference, pp. 2829-2831.

Famularo, D., Abdallah, C. T., Jadbabaie, A., Dorato, P. and Haddad, W. H. (1998). "Robust non-fragile LQ controllers: the static feedback case," Proc. of American Control Conference, pp. 110-1113.

Gard, T. C. (1988). Introduction to Stochastic Differential Equations, Marcel Dekker, New York.

Jadbabaie, A., Abdallah, C. T., Famularo, D., and Dorato, P. (1998). "Robust, non-fragile and optimal controller design via linear matrix inequalities," Proc. of American Control Conference, pp. 2832-2836.

Jeong, C. S., Yaz, E. E., and Yaz, Y. I. (2007). “An LMI Approach to Continuous-Time Observer Design with Stochastic Resilience," Proceedings of the American Control Conference, pp. 312-316.

Jeong, C. S., Yaz, E. E., and Yaz, Y. I. (2008). "LyapunovBased Design of Resilient Observers for a Class of Nonlinear Systems and General Performance Criteria," 2008 IEEE Multi-conference on Systems and Control, pp. 942-947.
Keel, L. H. and Bhattacharyya, S. P. (1997). "Robust, fragile, or optimal?" IEEE Trans. on Automat. Control, vol. 42, pp. $1098-1105$.

Keel, L. H. and Bhattacharyya, S. P. (1998). "Stability margins and digital implementation of controllers," Proc. of American Control Conference, pp. 2852-2856.

Mahmoud. M. S. (2004). "Resilient linear filtering of uncertain systems," Automatica, vol. 40, pp.1797-1802.

Takabashi, R.H.C., Dutra, D.A., Palhares, R.M., and Peres, P.K.D. (2000). "On Robust non-fragile static statefeedback controller synthesis," Proc. of the $39^{\text {th }}$ IEEE Conference on Decision and Control, vol. 5, pp. 49094914.

Yang, G.-H. and Wang., J. L. (2001). "Robust nonfragile Kalman filtering for uncertain linear systems," IEEE Transactions on Automatic Control, vol. 46, pp. 343-348.

Yaz, E. E., Jeong, C. S., and Yaz, Y. I. (2006). "An LMI approach to discrete-time observer design with stochastic resilience," J. of Computational \& Applied Math, vol. 188, pp 246-255.

Yaz, E. E. (1995). Robust Stability of Discrete-Time Randomly Perturbed Systems, Control and Dynamic Systems, vol. 73, pp. 89-120, Academic Press. 